\section{P75 SADDLE EMBOLISM INDUCED SYNCOPE: A CASE REPORT}

1,2Shauna Quinn*, 'Vicki Sandys, 'Deirdre O'Riordan. 'Trinity College Dublin, Dublin, Ireland; ${ }^{2}$ St James Hospital, Dublin, Ireland

10.1136/archdischild-2019-epa.430

Introduction Saddle pulmonary embolism is a life-threatening, clinically challenging diagnosis. Syncope is an uncommon presentation, which may be misdiagnosed. We report the case of a 17-year-old Caucasian girl with saddle embolism induced syncope.

Case presentation A 17-year-old girl, with no history of disease, was admitted to the emergency department following a 1 day history of two syncopal episodes on a background of left groin pain. She had been taking the COCP for 6 months for the treatment of acne. On admission, she became haemodynamically unstable with a BP 70/40, sinus tachy $150 \mathrm{bpm}$ and $83 \%$ oxygen saturations on room air. ECG demonstrated sinus tachy, RBBB and V1Q3. Doppler was positive for a left iliofemoral DVT. Urgent CTPA confirmed a massive saddle embolus, multiple right lower zone $\mathrm{PE}$ and increased right heart pressure. She was immediately transferred to IR where she had catheter-directed mechanical thrombolysis with $10 \mathrm{mg}$ TPA bolus, $500 \mathrm{IU}$ of heparin followed by an infusion of TPA $0.5 \mathrm{mg} /$ hour. Follow-up pulmonary angiogram demonstrated marked interval improvement with no evidence of residual thrombus. An ECHO was performed showing normal LV systolic function and a negative bubble study. Her lupus screen was negative. She was treated with Heparin according to the SJH protocol and discharged on Apixaban with haematology follow-up.

Discussion The annual incidence of paediatric PE is $0.5 /$ 10,000. Diagnosis may be delayed in young individuals, with some reporting a median of 7 days from presentation to diagnosis. Prompt diagnosis is vital to broaden management options and decrease mortality. This is the case of a young woman presenting with a clear indication for thrombolysis. Correct recognition and appropriate use of catheter directed thrombolysis was life-saving. Research indicates that it may be considered in patients with persistent haemodynamic instability, those at risk of death before systemic therapy can be effective and those with high bleeding risks.

Conclusion Pulmonary embolism is an important differential in patients presenting with syncope, with many of these having a large or saddle embolus. Early identification is vital to avoid haemodynamic compromise and to optimise survival.

\section{P76 NEONATAL SEPSIS? OR IS THERE SOMETHING MORE SERIOUS WE MIGHT BE MISSING?}

Liqa ur Rehman*, Paraic Curran. Portiuncula University Hospital, Ballinasloe, Ireland

\subsection{6/archdischild-2019-epa.431}

Aim To report a rare case of 6 weeks-old-infant with Severe Diabetic-Keto-Acidosis (DKA), a life threatening complication of Neonatal Diabetes Mellitus

Case history A 6 week-old-baby girl was brought in to the hospital by ambulance with an acute onset of lethargy, irritability, vomiting and breathlessness.
Further questioning with mother revealed large wet nappies, bad nappy rash and oral thrush going on for last 5 weeks.In addition she lost 800 gm weight in 24-48 hours before arrival.

Physical examination revealed; an unwell, emaciated and pale looking baby with signs of severe dehydration, who was catching her breath with each cry.Vitals were: HR 190/min,RR 70/min,BP 103/70(82), Spo2 90\%RA, Temp36.3c.

Systemic Examination was unremarkable apart from moderate increase work of breathing with bilateral equal air entry

Initial impression was late onset neonatal sepsis or gastroenteritis with severe dehydration.

Management Her airway was stabilized on Airvo support (FI02 50\%,flow $6 \mathrm{~L} / \mathrm{min}$ )

Two IV lines were inserted with full septic screening.She was given $10 \mathrm{ml} / \mathrm{kg}$ IV normal saline bolus over $10 \mathrm{mins}$ and covered with Empiric antimicrobial therapy.

Heal prick blood glucose: High, Ketones: 7

First Venous gas after 10 mins of arrival, confirmed severe DKA: VBG-3.15pm

\begin{tabular}{llllllll}
\hline $\mathrm{PH}$ & $\mathrm{PCO2}$ & $\mathrm{HCO}$ & $\mathrm{BE}$ & Lactate & Glucose & $\mathrm{Na}+$ & $\mathrm{K}+$ \\
\hline 6.8 & 2.8 & 4 & -28 & 4 & $>47$ & 150 & 6.7 \\
\hline
\end{tabular}

She was resuscitated further on NATIONAL-DKA-PROTOCOL under the expertise of the tertiary care team for five hours until she was transferred safely to PICU for further management.

Subsequent Capillary-Blood-Gases(CBG):

$\mathrm{CBG}-5 \mathrm{pm}$

\begin{tabular}{llllllll}
\hline $\mathrm{PH}$ & $\mathrm{PCO2}$ & $\mathrm{HCO} 3$ & $\mathrm{BE}$ & Lactate & Glucose & $\mathrm{Na}+$ & $\mathrm{K}+$ \\
\hline 6.98 & 2.78 & 3 & -27.5 & 3 & $>38.5$ & 148 & 6.0 \\
\hline
\end{tabular}

$\mathrm{CBG}-7.30 \mathrm{pm}$

\begin{tabular}{llllllll}
\hline $\mathrm{PH}$ & $\mathrm{PCO2}$ & $\mathrm{HCO} 3$ & $\mathrm{BE}$ & Lactate & Glucose & $\mathrm{Na}+$ & $\mathrm{K}+$ \\
\hline 7.06 & 2.27 & 4.9 & -25.4 & 2.82 & $>38.5$ & 145 & 4.5 \\
\hline
\end{tabular}

All other labs including blood culture were normal.

Outcome She stayed in PICU for three days and was transferred back home seven weeks later with no complications. She was started on insulin pump initially for five weeks until genetic results revealed her to be carrying heterogeneous mutations on KCNJ11 gene,confirming Permanent-NeonatalDiabetes-Mallitus. She was given a trail of sulfonylurea (Glibenclamide) which she tolerated well to obtain an optimal control of her blood sugars.

Conclusion Neonatal Diabetes is a rare condition however one should have a high index of suspicion in infants presenting with unusual weight loss, persistent refractory nappy rash and 
features of sepsis.Diagnosing DKA in this age group is crucial as any delay in initiating right treatment may result in significant morbidity and mortality.

\section{P77 REYE-LIKE SYNDROME AND SEVERE CARNITINE DEFICIENCY IN A BOY WITH AUTISM SPECTRUM DISORDER AND RESTRICTED DIET}

Lydia Healy*, Eimear Forbes, Jane Rice, Jane Leonard, Ellen Crushell. Temple Street Children's University Hospital, Dublin, Ireland

\subsection{6/archdischild-2019-epa.432}

Case Description A 9-year old boy presented to the Emergency Department with an acute history of vomiting, ataxia and reduced consciousness in the setting of a respiratory illness. In the two months prior he had intermittent episodes of vomiting, without headaches or visual disturbance.

On examination, he was encephalopathic (GCS 9/15), with marked hepatomegaly. Height and weight were on the $0.4^{\text {th }}$ $2^{\text {nd }}$ percentile and $9^{\text {th }}$ percentiles respectively.

$\mathrm{He}$ had a background of high-functioning autism spectrum disorder (ASD) and a long history (since aged 18 months) of rigid eating behaviour, with a diet restricted to corn-based crisps ('Rancheros'), rich-tea biscuits (2-3/day), French-fries and Coca-Cola (10\% glucose, drinking $1 \mathrm{~L} /$ day). He had recently commenced a multivitamin preparation.

Blood-work showed anaemia, elevated hepatic transaminases and creatinine kinase $(6000 \mathrm{U} / \mathrm{l})$, low albumin, hypoglycaemia (2.7 $\mathrm{mmol} / \mathrm{l})$ and deranged electrolytes. CT brain showed moderate, communicating hydrocephalus without pressure effects. An extra-ventricular drain was inserted but later removed as this was deemed to be a coincidental finding of arrested congenital hydrocephalus.

Management and Investigation $\mathrm{He}$ required intubation and ventilation for respiratory compromise (RSV infection). Given the Reye-like scenario and abnormal biochemistry, a metabolic disorder was suspected. Acylcarnitine profile showed undetectable free carnitine and amino acid levels were low, as were vitamin levels. He received carnitine supplementation and very cautious nasogastric feeding; biochemistry and neurological status improved. Extensive investigations including carnitine uptake studies did not reveal an inherited metabolic disorder.

Detailed nutritional analysis of the patient's diet revealed very low daily protein $(0.37 \mathrm{~g} / \mathrm{kg} /$ day $)$ and fat $(0.77 \mathrm{~g} / \mathrm{kg} /$ day $)$ but with normal total daily caloric intake of $1200 \mathrm{kCal}$, indicative of protein-energy-malnutrition (Kwashiorkor). He received intensive inpatient dietetic and psychological input and by discharge from hospital he was consuming an expanded range of foodstuffs. Carnitine is synthesised from essential amino acids lysine and methionine, but primarily it is obtained directly from protein in the diet. Cereal-based diets such as above are particularly low in the amino acids methionine and lysine.

Diagnosis and Follow-up On follow-up 2 years later, carnitine levels remain normal without supplementation. Protein intake is normal and diet is varied. Vertical growth has improved significantly with height now on the $91^{\text {st }}$ centile and hepatomegaly has resolved. The final diagnosis was dietary proteinenergy-malnutrition with profound secondary carnitine deficiency resulting in Reye-like syndrome.

This case reminds us that severe malnutrition exists in the developed world, and emphasises the need for detailed nutritional assessment of children on self-restricted diets, especially those with ASD.

\section{P78 AN UNUSUAL CAUSE OF AN ACUTE CONFUSIONAL STATE IN AN ADOLESCENT; TRANSIENT GLOBAL AMNESIA}

Alwyn Charles*, Ronan Callanan, Shozab Taj, Eoin Fitzgerald, Elizabeth O'Mahony, AnnMarie Murphy. Department of Paediatrics, UHL, Limerick, Ireland

\subsection{6/archdischild-2019-epa.433}

Background Transient global amnesia (TGA) is a syndrome that has been known to occur predominantly within the adult population. It's an entity characterised by sudden loss of memory with confusion that lasts less than 24 hours. The event itself is never associated with focal neurological findings and the patient remains conscious throughout the event. Some of the precipitants documented within the literature have included swimming, immersion in cold water, extreme physical exertion or emotional stress. There has been much debate to date over the etiology/pathogenesis of TGA, including migraine, epilepsy, emboli, ischaemia of hippocampal regions or even venous congestion have all been suggested causes. While TGA is more prevalent amongst those $>60$ years of age, there has been very few instances of TGA in childhood.

Aim To describe a case of transient global amnesia in a teenage boy.

Methods We report the clinical presentation, results of investigations and outcome to date in a fourteen year old boy who presented in an acute confusional state, the ultimate cause for which was transient global amnesia.

Results A 14 year old boy presented to the Paediatric Emergency Department (PED) for evaluation of acute confusion following a hurling match. A previously well, neurodevelopmentally appropriate child, he had no significant family history nor did he have any regular medications or allergies.

Being an active sportsman, he had just participated in an intense hurling match that had lasted almost two hours in exceptionally cold weather. On cessation of the game, he had approached his coach disoriented and claiming ' $\mathrm{I}$ do not know where I am or why I am here'.

He continued to ask bizarre repetitive questions, and had no memory of the match for the following 12 hours. His neurological exam was normal, with no loss of consciousness or head injury. Basic blood work, toxicology screen, CXR and ECG were normal. Twenty fours later he had completely recovered. CT brain, MR brain with angiography and EEG were normal.

At follow-up six weeks later, he had remained perfectly well with normal neurological assessment.

Conclusion Childhood transient global amnesia is rare. Recurrence has been reported in those with a history of recurrent migraine. Our case is interesting in that it deviates from such reports whereby this episode seems solely provoked by Valsalva-like activity (extreme exertion)

\section{P79 KAWASAKI DISEASE}

Zachary Tan*, Irina Chistol, Samira Mohamed, Salma Mohamed, Nurrasyidah Abdul Halim, Victor Morris. St Luke's Hospital, Kilkenny, Ireland

\subsection{6/archdischild-2019-epa.434}

Introduction Kawasaki Disease (KD) is a relatively rare disease with a reported current, annual incidence in Europe of about $5-10 / 100,000$ children younger than 5 years_. The highest 